\title{
UNIFORM TIGHTNESS FOR TRANSITION PROBABILITIES
}

\author{
JUN KAWABE
}

\begin{abstract}
The aim of this paper is to give a notion of uniform tightness for transition probabilities on topological spaces, which assures the uniform tightness of compound probability measures. Then the upper semicontinuity of set-valued mappings are used in essence. As an important example, the uniform tightness for Gaussian transition probabilities on the strong dual of a nuclear real Fréchet space is studied. It is also shown that some of our results contain well-known results concerning the uniform tightness and the weak convergence of probability measures.
\end{abstract}

\section{Introduction}

Let $X$ and $Y$ be topological spaces. In this paper, we present a notion of uniform tightness for transition probabilities on $X \times Y$ which assures the uniform tightness for compound probability measures $\mu \circ \lambda$ defined by

$$
\mu \circ \lambda(D)=\int_{X} \lambda\left(x, D_{x}\right) \mu(d x)
$$

for a measure $\mu$ on $X$ and a transition probability $\lambda$ on $X \times Y$. We may consider that the compound probability measure is a generalization of the product measure or the convolution measure, and have to notice that the weak convergence of convolution measures has been looked into in great details by Csiszár [2, 3] and Kallianpur [6]. In Section 2 we recall notation and necessary definitions and results concerning probability measures on topological spaces, and then give a necessary and sufficient condition for a probability measure-valued mapping to be a transition probability in terms of the measurability of its characteristic functional.

Received February 18, 1994; revised September 4, 1995.

1991 Mathematics Subject Classification. Primary 60B05, 60B10, 60B11.

Key words and phrases. Transition probability, compound probability measure, upper semicontinuous, set-valued mapping, uniform tighness, Gaussian, nuclear space.

Research supported by Grant-in-Aid for General Scientific Research No. 07640189, the Ministry of Education, Science, Sports and Culture, Japan. 
In Section 3 we present a notion of uniform tightness for transition probabilities, using the upper semicontinuity of set-valued mappings, so that the corresponding set of compound probability measures is uniformly tight. We also give a sufficient condition for the weak convergence of a net of compound probability measures.

In Section 4 we study Gaussian transition probabilities on the strong dual of a nuclear real Fréchet space as an important example of the uniform tightness for transition probabilities. We also show that some of the results in this section contain well-known results concerning the uniform tightness and the weak convergence of probability measures.

Throughout this paper, we suppose that all the topological spaces and all the topological linear spaces are Hausdorff.

\section{Preliminaries}

Let $(X, \mathcal{A})$ be a measurable space and $Y$ a topological space. We denote by $\mathcal{B}(Y)$ the $\sigma$-algebra of all Borel subsets of $Y$. By a Borel measure on $Y$ we mean a finite measure defined on $\mathcal{B}(Y)$ and we denote by $\mathcal{P}(Y)$ the set of all Borel probability measures on $Y$.

If $Y$ is completely regular, we equip $\mathcal{P}(Y)$ with the weakest topology for which the functionals

$$
\nu \in \mathcal{P}(Y) \mapsto \int_{Y} g(y) \nu(d y), \quad g \in C_{b}(Y)
$$

are continuous. Here $C_{b}(Y)$ denotes the set of all bounded continuous real-valued functions on $Y$. This topology on $\mathcal{P}(Y)$ is called the weak topology, and we say that a net $\left\{\nu_{\alpha}\right\}$ in $\mathcal{P}(Y)$ converges weakly to a Borel probability measure $\nu$ and we write $\nu_{\alpha} \stackrel{w}{\rightarrow} \nu$, if

$$
\lim _{\alpha} \int_{Y} g(y) \nu_{\alpha}(d y)=\int_{Y} g(y) \nu(d y)
$$

for every $g \in C_{b}(Y)$.

A transition probability $\lambda$ on $X \times Y$ is defined to be a mapping from $X$ into $\mathcal{P}(Y)$ which satisfies

(T1) for every $B \in \mathcal{B}(Y)$, the function $x \in X \mapsto \lambda_{x}(B) \equiv \lambda(x, B)$ is measurable with respect to $\mathcal{A}$ and $\mathcal{B}(\mathbb{R})$.

In case $X$ is also a topological space we always take $\mathcal{A}=\mathcal{B}(X)$.

Denote by $C(Y)$ the set of all continuous real-valued functions on $Y$. For each transition probability $\lambda$ on $X \times Y$ and each $h \in C(Y)$, we can define a measurable function

$$
x \in X \mapsto \chi[\lambda, h](x) \equiv \int_{Y} e^{i h(y)} \lambda(x, d y) .
$$

In the rest of this section we give a condition for a mapping $\lambda$ from $X$ into $\mathcal{P}(Y)$ to be a transition probability on $X \times Y$ in terms of the measurability of the above function $\chi[\lambda, h](x)$. Denote by $\mathbb{R}^{N}$ be the $N$-dimensional Euclidian space. For $u=$ 
$\left(u_{1}, u_{2}, \ldots, u_{N}\right), v=\left(v_{1}, v_{2}, \ldots, v_{N}\right) \in \mathbb{R}^{N}$, we set $\langle u, v\rangle=u_{1} v_{1}+u_{2} v_{2}+\cdots+u_{N} v_{N}$ and $\|u\|=\sqrt{\langle u, u\rangle}$. We denote by $K\left(\mathbb{R}^{N}\right)$ the set of all continuous complex-valued functions on $\mathbb{R}^{N}$ with compact supports.

Lemma 1. Let $(X, \mathcal{A})$ be a measurable space and let $\lambda$ be a mapping from $X$ into $\mathcal{P}\left(\mathbb{R}^{N}\right)$. Then $\lambda$ is a transition probability on $X \times \mathbb{R}^{N}$ if and only if for each $u=\left(u_{1}, u_{2}, \ldots, u_{N}\right) \in \mathbb{R}^{N}$, the function

$$
x \in X \mapsto \hat{\lambda}_{x}(u) \equiv \int_{\mathbb{R}^{N}} e^{i(u, v)} \lambda(x, d v)
$$

is measurable.

Proof. The only if part is obvious and so we prove if part. Since a family of sets $B \in \mathcal{B}(Y)$ for which the functions $x \in X \mapsto \lambda(x, B)$ are measurable is a Dynkin system (see Theorem 4.1.2 of Ash [1]; Dynkin System Theorem), it suffices to show that for each open subset $G$ of $\mathbb{R}^{N}$, the function $x \in X \mapsto \lambda(x, G)$ is measurable.

Let $G$ be an open subset of $\mathbb{R}^{N}$. Then we can find a sequence $\left\{g_{n}\right\}$ of real-valued, increasing functions in $K\left(\mathbb{R}^{N}\right)$ such that $1_{G}(u)=\sup _{n \geq 1} g_{n}(u)$ for all $u \in \mathbb{R}^{N}$. Consequently by monotone convergence theorem we have

$$
\lambda(x, G)=\lim _{n \rightarrow \infty} \int_{\mathbb{R}^{N}} g_{n}(u) \lambda(x, d u) \text { for all } x \in X,
$$

and from this we have only to show that for each $g \in K\left(\mathbb{R}^{N}\right)$, the function $x \in X \rightarrow$ $\int_{\mathbb{R}^{N}} g(u) \lambda(x, d u)$ is measurable.

Fix $g \in K\left(\mathbb{R}^{N}\right)$. Then we can find a sequence $\left\{g_{n}\right\}$ of infinitely differentiable complex-valued functions on $\mathbb{R}^{N}$ with compact supports such that

$$
\sup _{u \in \mathbb{R}^{N}}\left|g(u)-g_{n}(u)\right| \rightarrow 0 \quad \text { as } \quad n \rightarrow \infty
$$

(see Yosida [15; Proposition I.1.8]). Since the Fourier transform $\mathcal{F}: \mathcal{S}\left(\mathbb{R}^{N}\right) \rightarrow \mathcal{S}\left(\mathbb{R}^{N}\right)$ is surjective, where $\mathcal{S}\left(\mathbb{R}^{N}\right)$ denotes the set of all rapidly decreasing complex-valued functions on $\mathbb{R}^{N}$ (see [15; Theorem VI.1.1]), there exists a sequence $\left\{h_{n}\right\}$ of functions in $\mathcal{S}\left(\mathbb{R}^{N}\right)$ such that for each $n \geq 1$,

$$
g_{n}(u)=\left(\mathcal{F} h_{n}\right)(u) \equiv(2 \pi)^{-N / 2} \int_{\mathbb{R}^{N}} e^{i<u, v>} h_{n}(v) d v \text { for all } u \in \mathbb{R}^{N} .
$$

Consequently for each $x \in X$ we have

$$
\begin{aligned}
\int_{\mathbb{R}^{N}} g(u) \lambda(x, d u) & =(2 \pi)^{-N / 2} \lim _{n \rightarrow \infty} \int_{\mathbb{R}^{N}} \int_{\mathbb{R}^{N}} e^{i<u, v>} h_{n}(v) d v \lambda(x, d u) \\
& =(2 \pi)^{-N / 2} \lim _{n \rightarrow \infty} \int_{\mathbb{R}^{N}} \hat{\lambda}_{x}(v) h_{n}(v) d v .
\end{aligned}
$$


Put $\varphi(x, v)=\hat{\lambda}(v)$ for all $x \in X$ and $v \in \mathbb{R}^{N}$. Then for each $v \in \mathbb{R}^{N}, \varphi(\cdot, v)$ is measurable by assumption, and for each $x \in X, \varphi(x, \cdot)$ is continuous on $\mathbb{R}^{N}$ since the characteristic function of a probability measure on $\mathcal{B}\left(\mathbb{R}^{N}\right)$ is continuous on $\mathbb{R}^{N}$. Therefore by Klein and Thompson [8; Lemma 13.2.3], $\varphi$ is $\mathcal{A} \times \mathcal{B}\left(\mathbb{R}^{N}\right)$-measurable, and this, together with Fubini's theorem, implies the measurability of the function $x \in X \mapsto \int_{\mathbb{R}^{N}} \hat{\lambda}_{x}(v) h_{n}(v) d v$ for each $n \geq 1$. Thus the function $x \in X \mapsto \int_{\mathbb{R}^{N}} g(u) \lambda(x, d u)$ is measurable and the proof is complete.

Recall that a topological space is called a Suslin space if it is the continuous image of some Polish space and recall that a subset $H$ of $C(Y)$ is said to separate points of $Y$ if for each $y_{1}, y_{2} \in Y$ with $y_{1} \neq y_{2}$, there exists a function $h \in H$ such that $h\left(y_{1}\right) \neq h\left(y_{2}\right)$.

Proposition 1. Let $(X, \mathcal{A})$ be a measurable space and $Y$ a completely regular Suslin space, and let $\lambda$ be a mapping from $X$ into $\mathcal{P}(Y)$. Assume that a linear subspace $H$ of $C(Y)$ separates points of $Y$. Then $\lambda$ is a transition probability on $X \times Y$ if and only if for each $h \in H$, the function $x \in X \mapsto \chi[\lambda, h](x)$ is measurable.

Proof. The only if part is obvious and so we prove if part. Let us first notice that $\mathcal{B}(Y)$ is generated by the cylinder sets of the form

$$
C=\left\{y \in Y:\left(h_{1}(y), h_{2}(y), \cdots, h_{N}(y)\right) \in B\right\}
$$

where $h_{1}, h_{2}, \ldots, h_{N} \in H$ and $B \in \mathcal{B}\left(\mathbb{R}^{N}\right)$ (see Theorem I.1.2 of Vakhania et al. [14]). There this result is proved when $Y$ is a Polish space. However the proof works equally well when $Y$ is a Suslin space since $\mathcal{B}(Y)$ concides with the $\sigma$-algebra generated by a sequence of real-valued Borel measurable functions on a Suslin space $Y$ separating points of $Y$, and every open cover of any open subset of a Suslin space has a countable subcover; see Schwartz [12], Lemma 18 of page 108 and Proposition 3 of page 104. Consequently it is sufficient to show that for each cylinder set $C$ of the above form, the function $x \in X \mapsto \lambda(x, C)$ is measurable, since a family of sets $C \in \mathcal{B}(Y)$ for which the function $x \in X \mapsto \lambda(x, C)$ are measurable is a Dynkin system. Thus we prove that for each $B \in \mathcal{B}\left(\mathbb{R}^{N}\right)$, the function $x \in X \mapsto \gamma_{x}(B) \equiv \lambda_{x} \circ \varphi^{-1}(B)$ is measurable, where $\varphi(y)=\left(h_{1}(y), h_{2}(y), \ldots, h_{N}(y)\right), y \in Y$.

For each $u=\left(u_{1}, u_{2}, \ldots, u_{N}\right) \in \mathbb{R}^{N}$, we have

$$
\hat{\gamma}_{x}(u)=\int_{Y} e^{i\left\{u_{1} h_{1}(y)+u_{2} h_{2}(y)+\cdots+u_{n} h_{N}(y)\right\}} \lambda(x, d y)=\chi\left[\lambda, \sum_{j=1}^{N} u_{j} h_{j}\right](x)
$$

and hence for each $u \in \mathbb{R}^{N}$, the function $x \in X \mapsto \hat{\gamma}_{x}(u)$ is measurable by assumption. Therefore from Lemma 1 the measurability of the function $x \in X \mapsto \gamma_{x}(B)$ follows. 


\section{Uniform Tightness for Transition Probabilities}

Let $X$ and $Y$ be topological spaces. Let us denote by $\mathcal{T}(X, Y)$ the set of all transition probabilities on $X \times Y$ and denote by $\mathcal{T}^{*}(X, Y)$ the set of all $\lambda \in \mathcal{T}(X, Y)$ which satisfy the condition

(T2) for each $D \in \mathcal{B}(X \times Y)$, the function $x \in X \mapsto \lambda\left(x, D_{x}\right)$ is Borel measurable. Here for a subset $D$ of $X \times Y$ and $x \in X, D_{x}$ denotes the section determined by $x$, that is, $D_{x}=\{y \in Y:(x, y) \in D\}$.

Let $\mu \in \mathcal{P}(X)$ and $\lambda \in \mathcal{T}^{*}(X, Y)$. Then we can define a Borel probability measure $\mu \circ \lambda$ on $X \times Y$, which is called the compound probability measure of $\mu$ and $\lambda$, by

$$
\mu \circ \lambda(D)=\int_{X} \lambda\left(x, D_{x}\right) \mu(d x) \text { for all } D \in \mathcal{B}(X \times Y)
$$

Denote by $\mu \lambda$ the projection of $\mu \circ \lambda$ onto $Y$, that is, $\mu \lambda(B)=\mu \circ \lambda(X \times B)$ for all $B \in \mathcal{B}(Y)$. By a standard argument, we can show that the Fubini's theorem remains valid for all Borel measurable and $\mu \circ \lambda$-integrable functions $f$ on $X \times Y$;

$$
\int_{X \times Y} f(x, y) \mu \circ \lambda(d x, d y)=\int_{X} \int_{Y} f(x, y) \lambda(x, d y) \mu(d x) .
$$

It is obvious that (T2) implies (T1), and (T2) is satisfied, for instance, if the product $\sigma$-algebra $\mathcal{B}(X) \times \mathcal{B}(Y)$ coincides with $\mathcal{B}(X \times Y)$ (this is satisfied if $X$ and $Y$ are Suslin spaces; see [12], page 105). We also know that (T2) is satisfied for any continuous $\tau$-smooth transition probability on an arbitrary topological space (see Proposition 1 of Kawabe [7]). In what follows, for $P \subset \mathcal{P}(X)$ and $Q \subset \mathcal{T}^{*}(X, Y)$, we set $P \circ Q=\{\mu \circ \lambda$ : $\mu \in P$ and $\lambda \in Q\}$ and $P Q=\{\mu \lambda: \mu \in P$ and $\lambda \in Q\}$.

Recall that a subset $P$ of $\mathcal{P}(X)$ is said to be uniformly tight if for each $\varepsilon>0$, there exists a compact subset $K_{\varepsilon}$ of $X$ such that

$$
\mu\left(X-K_{\varepsilon}\right)<\varepsilon \quad \text { for all } \quad \mu \in P
$$

(see Prokhorov [10]). It is easy to see that $P \circ Q$ is uniformly tight if and only if $P$ and $P Q$ are uniformly tight. However $P Q$ and $P \circ Q$ are not necessarily uniformly tight even if $P$ is uniformly tight and $Q[x]=\left\{\lambda_{x}: \lambda \in Q\right\}$ is uniformly tight for each $x \in X$ as is seen in the following example. In what follows, $\delta_{x}$ denotes the Dirac measure concentrated on $x$, that is, $\delta_{x}(B)=1$ if $x \in B ; \delta_{x}(B)=0$ if $x \notin B$.

Example. Let $X=Y=\mathbb{R}$. For each $n \geq 1$, put

$$
s_{n}^{2}(x)= \begin{cases}0 & \text { for } x \leq 0 \\ n^{2} x & \text { for } 0<x \leq 1 / n \\ 2 n-n^{2} x & \text { for } 1 / n<x \leq 2 / n \\ 0 & \text { for } 2 / n<x\end{cases}
$$


and define a transition probability $\lambda_{n}$ on $\mathbb{R} \times \mathbb{R}$ by $\lambda_{n}(x, \cdot)=\mathcal{N}\left[0, s_{n}^{2}(x)\right]$, where $\mathcal{N}\left[m, \sigma^{2}\right]$ denotes the Gaussian measure on $\mathbb{R}$ with the mean $m$ and the variance $\sigma^{2}$. We also put $P=\left\{\delta_{1 / n}\right\}$ and $Q=\left\{\lambda_{n}\right\}$. Then $P$ is uniformly tight and $Q[x]$ is uniformly tight for each $x \in \mathbb{R}$, but $P Q$ and $P \circ Q$ are not uniformly tight.

Proof. It follows from Lemma 1 that the $\lambda_{n}$ 's are transition probabilities on $\mathbb{R} \times \mathbb{R}$. Clearly $P$ is uniformly tight and for each $x \in \mathbb{R}, Q[x]$ is also uniformly tight since $\sup _{n \geq 1} s_{n}^{2}(x)<\infty$.

We now note that a subset $A$ of $\mathcal{P}\left(\mathbb{R}^{N}\right)$ is uniformly tight if and only if the set $\{\hat{\mu}: \mu \in A\}$ of characteristic functions of $\mu$ defined by

$$
\hat{\mu}\left(p_{1}, p_{2}, \cdots, p_{N}\right)=\int_{\mathbb{R}^{N}} e^{i \sum_{j=1}^{N} p_{j} u_{j}} \mu\left(d u_{1}, d u_{2}, \cdots, d u_{N}\right)
$$

for each $p=\left(p_{1}, p_{2}, \cdots, p_{N}\right) \in \mathbb{R}^{N}$, is equicontinuous at $p=0 \in \mathbb{R}^{N}$. Since $\left(\delta_{1 / n} \lambda_{n}\right)^{\wedge}(q)$ $=e^{-q^{2} n / 2}$ and $\left(\delta_{1 / n} \circ \lambda_{n}\right)^{\wedge}(p, q)=e^{i p / n-q^{2} n / 2}$ for all $p, q \in \mathbb{R},\left\{\left(\delta_{1 / n} \lambda_{n}\right)^{\wedge}\right\}$ and $\left\{\left(\delta_{1 / n} \circ\right.\right.$ $\left.\left.\lambda_{n}\right)^{\wedge}\right\}$ are not equicontinuous at $q=0$ and at $(p, q)=(0,0)$, respectively. Therefore $P Q$ and $P \circ Q$ are not uniformly tight.

We now present a notion of uniform tightness for transition probabilities. We say that $Q \subset \mathcal{T}(X, Y)$ is uniformly tight if each $\varepsilon>0$, we can find an upper semicontinuous compact-valued mapping $\Lambda_{\varepsilon}: X \rightarrow Y$ such that

$$
\lambda\left(x, Y-\Lambda_{\varepsilon}(x)\right)<\varepsilon
$$

for all $x \in X$ and $\lambda \in Q$. Recall that a set-valued mapping $\Lambda: X \rightarrow Y$ is upper semicontinuous if $\Lambda^{w}(F) \equiv\{x \in X: \Lambda(x) \cap F \neq \varnothing\}$ is a closed subset of $X$ for every closed subset $F$ of $Y$. For the reader's convenience, we collect some well-known facts about upper semicontinuous set-valued mappings which will be used later (see [8], pages 89 and 90).

Proposition 2. Let $\Gamma$ and $\Lambda$ be upper semicontinuous set-valued mappings from $X$ to $Y$. Then one has:

(1) If $\Lambda$ is compact-valued then $\Lambda(K)=\cup_{x \in K} \Lambda(x)$ is compact for every compact subset $K$ of $X$.

(2) If $Y$ is a topological linear space, and $\Gamma$ and $\Lambda$ are compact-valued, then the mapping $x \in X \mapsto \Gamma(x)+\Lambda(x)$ is compact-valued and upper semicontinuous.

The following theorem asserts that our notion of uniform tightness for transition probabilities assures the uniform tightness of compound probability measures.

Theorem 1. Let $X$ and $Y$ be topological spaces. If $P \subset \mathcal{P}(X)$ and $Q \subset$ $\mathcal{T}^{*}(X, Y)$ are uniformly tight, then $P \circ Q \subset \mathcal{P}(X \times Y)$ is uniformly tight. 
Proof. Fix $\varepsilon>0$. Since $P$ and $Q$ are uniformly tight, we can find a compact subset $K_{\varepsilon}$ of $X$ and an upper semicontinuous compact-valued mapping $\Lambda_{\varepsilon}: X \rightarrow Y$ such that

$$
\mu\left(K_{\varepsilon}\right) \geq 1-\varepsilon \quad \text { and } \quad \lambda\left(x, \Lambda_{\varepsilon}(x)\right) \geq 1-\varepsilon .
$$

Since $L_{\varepsilon}=\cup_{x \in K_{\varepsilon}} \Lambda_{\varepsilon}(x)$ is a compact subset of $Y$ by (1) of Proposition 2, we have

$$
\mu \circ \lambda\left(K_{\varepsilon} \times L_{\varepsilon}\right)=\int_{K_{\varepsilon}} \lambda\left(x, L_{\varepsilon}\right) \mu(d x) \geq \int_{K_{\varepsilon}} \lambda\left(x, \Lambda_{\varepsilon}(x)\right) \mu(d x) \geq(1-\varepsilon)^{2},
$$

and this implies the uniform tightness of $P \circ Q$.

Let $X$ be a topological space. Denote by $C(X)$ the set of all continuous real-valued functions on $X$. We say that a subset $F$ of $C(X)$ is equicontinuous on a set $A$ of $X$ if the set of all restrictions of functions of $F$ to $A$ is equicontinuous on $A$.

A Borel measure $\mu$ on $X$ is said to be $\tau$-smooth if for every increasing net $\left\{G_{\alpha}\right\}$ of open subsets of $X$, we have $\mu\left(\cup_{\alpha} G_{\alpha}\right)=\sup _{\alpha} \mu\left(G_{\alpha}\right)$. Denote by $\mathcal{P}_{\tau}(X)$ the set of all $\tau$-smooth probability measures on $X$. Every Radon measure is tight and $\tau$-smooth, and if $X$ is regular, every $\tau$-smooth measure is regular (see [14], Proposition I.3.1). Conversely every tight and regular Borel measure is Radon. The proof of the following lemma is an easy modification of the proof of Theorem 2 in [2], and so we omit its proof.

Lemma 2. Let $X$ be a completely regular topological space and let $\left\{\mu_{\alpha}\right\}$ be a net in $\mathcal{P}(X)$ which is uniformly tight. Assume that a net $\left\{\varphi_{\alpha}\right\}$ in $C_{b}(X)$ satisfies

(a) $\left\{\varphi_{\alpha}\right\}$ is uniformly bounded;

(b) $\left\{\varphi_{\alpha}\right\}$ is equicontinuous on every compact subset of $X$.

If $\mu \in \mathcal{P}_{\tau}(X)$ and $\mu_{\alpha} \stackrel{w}{\rightarrow} \mu$, and if $\varphi \in C_{b}(X)$ and $\varphi_{\alpha}(x) \rightarrow \varphi(x)$ for each $x \in X$, then we have

$$
\lim _{\alpha} \int_{X} \varphi_{\alpha}(x) \mu_{\alpha}(d x)=\int_{X} \varphi(x) \mu(d x) .
$$

We give a sufficient condition for the weak convergence of a net of compound probability measures.

Theorem 2. Let $X$ and $Y$ be completely regular Suslin spaces. Let $H$ be a linear subspace of $C(Y)$ which separates points of $Y$. Assume that a net $\left\{\lambda_{\alpha}\right\}$ in $\mathcal{T}(X, Y)$ and $\lambda \in \mathcal{T}(X, Y)$ satisfy

(a) $\left\{\lambda_{\alpha}\right\}$ is uniformly tight;

(b) for each $h \in H$, the set $\left\{\chi\left[\lambda_{\alpha}, h\right]\right\}$ of the functions $x \in X \mapsto \chi\left[\lambda_{\alpha}, h\right](x)$ is equicontinuous on every compact subset of $X$;

(c) $\chi\left[\lambda_{\alpha}, h\right](x) \rightarrow \chi[\lambda, h](x)$ for each $x \in X$ and $h \in H$.

Then for any uniformly tight net $\left\{\mu_{\alpha}\right\}$ in $\mathcal{P}(X)$ converging weakly to $\mu \in \mathcal{P}(X)$, we have $\mu_{\alpha} \circ \lambda_{\alpha} \stackrel{w}{\rightarrow} \mu \circ \lambda$. 
Proof. Since $\left\{\mu_{\alpha}\right\}$ and $\left\{\lambda_{\alpha}\right\}$ are uniformly tight, $\left\{\mu_{\alpha} \circ \lambda_{\alpha}\right\}$ is also uniformly tight by Theorem 1 and the fact that $\mathcal{T}^{*}(X, Y)=\mathcal{T}(X, Y)$. Therefore it is a relatively compact subset of $\mathcal{P}(X \times Y)$.

For each $\gamma \in \mathcal{P}(X \times Y)$, let us define its characteristic function $\hat{\gamma}$ by

$$
\hat{\gamma}(f)=\int_{X \times Y} e^{i f(x, y)} \gamma(d x, d y), \quad f \in C(X \times Y) .
$$

Then by a standard argument (see [14], Theorem IV.3.1), it is sufficient to show that for each $g \in C(X)$ and $h \in H$, we have $\left(\mu_{\alpha} \circ \lambda_{\alpha}\right)^{\wedge}(g \oplus h) \rightarrow(\mu \circ \lambda)^{\wedge}(g \oplus h)$, since $C(X) \oplus H=\{g \oplus h: g \in C(X), h \in H\}$ is a linear subspace of $C(X \times Y)$ which separates points of $X \times Y$, where $(g \oplus h)(x, y)=g(x)+h(y),(x, y) \in X \times Y$. that

Fix $g \in C(X)$ and $h \in H$. From Lemma 2 and assumptions (b) and (c) it follows

$$
\lim _{\alpha} \int_{X} e^{i g(x)} \chi\left[\lambda_{\alpha}, h\right](x) \mu_{\alpha}(d x)=\int_{X} e^{i g(x)} \chi[\lambda, h](x) \mu(d x),
$$

and this implies that $\left(\mu_{\alpha} \circ \lambda_{\alpha}\right)^{\wedge}(g \oplus h) \rightarrow(\mu \circ \lambda)^{\wedge}(g \oplus h)$.

We have typical and somewhat trivial examples of uniformly tight transition probabilities below. We study non-trivial examples in the following section.

Proposition 3. Let $X$ be a topological space and $Y$ a completely regular topological space.

(1) For each $\alpha$, put $\lambda_{\alpha}(x, B)=\nu_{\alpha}(B)$ for all $x \in X$ and $B \in \mathcal{B}(Y)$, where $\left\{\nu_{\alpha}\right\} \subset$ $\mathcal{P}_{\tau}(Y)$ is uniformly tight. Then the $\lambda_{\alpha}$ 's satisfy $(\mathrm{T} 2)$, and $\left\{\lambda_{\alpha}\right\}$ is uniformly tight.

(2) Let $X=Y=G$ be a topological group. For each $\alpha$, put $\lambda_{\alpha}(x, B)=\nu_{\alpha}\left(B x^{-1}\right)$ for all $x \in G$ and $B \in \mathcal{B}(G)$, where $\left\{\nu_{\alpha}\right\} \subset \mathcal{P}_{\tau}(G)$ is uniformly tight. Then the $\lambda_{\alpha}$ 's satisfy $(\mathrm{T} 2)$, and $\left\{\lambda_{\alpha}\right\}$ is uniformly tight.

Proof. (1) It follows from Lemma I.4.1 of [14] that the $\lambda_{\alpha}$ 's satisfy (T2), and the uniform tightness of $\left\{\lambda_{\alpha}\right\}$ is obvious.

(2) Let $\theta$ be a continuous mapping from $G \times G$ into $G$. For each $\alpha$, put

$$
\lambda_{\alpha}(x, B)=\nu_{\alpha}(\{y \in G: \theta(x, y) \in B\}) \quad \text { for all } \quad x \in G \text { and } B \in \mathcal{B}(G) .
$$

Note that (2) is a special case of this setting. The fact that the $\lambda_{\alpha}$ 's satisfy (T2) is an immediate consequence of Lemma I.4.1 of [14], together with the fact that for each Borel subset $B$ of $G \times G, \tilde{B} \equiv\{(x, y) \in G \times G:(x, \theta(x, y)) \in B\}$ is also a Borel subset of $G \times G$ and $[\tilde{B}]_{x}=\left\{y \in G: \theta(x, y) \in B_{x}\right\}$ for each $x \in G$. Hence we have only to prove that if $\left\{\nu_{\alpha}\right\}$ is uniformly tight, so is $\left\{\lambda_{\alpha}\right\}$.

For $\varepsilon>0$ there exists a compact subset $K_{\varepsilon}$ of $G$ such that $\nu_{\alpha}\left(G-K_{\varepsilon}\right)<\varepsilon$ for all $\alpha$. Put $\Lambda_{\varepsilon}(x)=\theta\left(x, K_{\varepsilon}\right)$ for all $x \in G$. Then $\Lambda_{\varepsilon}$ is clearly compact-valued mapping and 
it is upper semicontinuous by the continuity of $\theta$ and the compactness of $K_{\varepsilon}$. Moreover we have

$$
\lambda_{\alpha}\left(x, G-\Lambda_{\varepsilon}(x)\right)=\nu_{\alpha}\left(\left\{y \in G: \theta(x, y) \notin \theta\left(x, K_{\varepsilon}\right)\right\}\right) \leq \nu_{\alpha}\left(G-K_{\varepsilon}\right)<\varepsilon
$$

for all $x \in G$ and all $\alpha$. Therefore $\left\{\lambda_{\alpha}\right\}$ is uniformly tight.

\section{Gaussian Transition Probabilities on Nuclear Spaces}

In this section we study Gaussian transition probabilities on nuclear spaces, such as the strong dual of the space of all rapidly decreasing functions, which are important and non-trivial examples of uniformly tight transition probabilities.

Let $\Psi$ be a nuclear real Fréchet space, $\Psi^{\prime}$ the dual of $\Psi$ and $\langle\cdot, \cdot\rangle$ the bilinear form on $\Psi \times \Psi^{\prime}$. Let us denote by $\Psi_{\sigma}^{\prime}$ and $\Psi_{\beta}^{\prime}$ the weak and strong dual of $\Psi$ with the weak topology $\sigma\left(\Psi^{\prime}, \Psi\right)$ and the strong topology $\beta\left(\Psi^{\prime}, \Psi\right)$, respectively. For the following properties which $\Psi_{\beta}^{\prime}$ enjoys the reader will find more details and proofs in Schaefer [11] and Fernique [4].

\section{Proposition 4.}

(1) $\Psi_{\beta}^{\prime}$ is a Montel space, that is, it is a barreled space which every closed, bounded subset is compact.

(2) $\Psi_{\beta}^{\prime}$ is a completely regular Suslin space, in fact, Lusin space.

(3) Every closed, bounded subset of $\Psi_{\sigma}^{\prime}$ is a compact and sequentially compact subset of $\Psi_{\beta}^{\prime}$.

A seminorm $p$ on $\Psi$ is called Hilbertian $(H$-seminorm) if $p$ has the form $p(u)=$ $\sqrt{p(u, u)}$, where $p(u, v)$ is a symmetric, non-negative definite, bilinear functional on $\Psi \times \Psi$. Then the $p$-completion of $\Psi /$ ker $p$, denoted by $\Psi_{p}$, is a separable Hilbert space, and its dual $\Psi_{p}^{\prime}$ is also a separable Hilbert space with the norm $p^{\prime}(\eta)=\sup \{|\langle u, \eta\rangle|: p(u) \leq$ 1\}.

Let $p$ and $q$ be $H$-seminorms on $\Psi$. Following Itô [5], we say that $p$ is said to be bounded by $q$, written $p \prec q$, if

$$
(p: q)=\sup \{p(u): q(u) \leq 1\}<\infty .
$$

We also say that $p$ is said to be Hilbert-Schmidt bounded by $q$, written $p \prec_{H S} q$, if $p \prec q$ and

$$
(p: q)_{H S}=\left(\sum_{j=1}^{\infty} p\left(e_{j}\right)^{2}\right)^{1 / 2}<\infty \text { for some CONS }\left\{e_{j}\right\} \text { in }(\Psi, q) .
$$

It is well-known and is easily verified that $P \subset \mathcal{P}\left(\Psi_{\beta}^{\prime}\right)$ is uniformly tight if and only if for each $\varepsilon>0$, there exists a continuous $H$-seminorm $p_{\varepsilon}$ on $\Psi$ such that

$$
\mu\left(\left\{\eta \in \Psi^{\prime}:|<u, \eta>| \leq p_{\varepsilon}(u) \text { for all } \mu \in \Psi\right\}\right) \geq 1-\varepsilon
$$


for all $\mu \in P$. For the uniform tightness for transition probabilities we have:

Theorem 3. Let $X$ be a topological space which satisfies the first axiom of countability and $Q$ a subset of $\mathcal{T}\left(X, \Psi_{\beta}^{\prime}\right)$. Assume that for each $\varepsilon>0$ there exists a mapping $p_{\varepsilon}: X \times \Psi \rightarrow[0, \infty)$ satisfying $X$;

(a) for each $u \in \Psi$, the mapping $x \in X \mapsto p_{\varepsilon}(x, u)$ is upper semicontinuous on

(b) for each $x \in X, p_{\varepsilon}(x)(\cdot) \equiv p_{\varepsilon}(x, \cdot)$ is a continuous $H$-seminorm on $\Psi$;

(c) $\lambda\left(x,\left\{\eta \in \Psi^{\prime}:|\langle u, \eta\rangle| \leq p_{\varepsilon}(x, u)\right.\right.$ for all $\left.\left.u \in \Psi\right\}\right) \geq 1-\varepsilon$ for all $x \in X$ and $\lambda \in Q$. Then $Q$ is uniformly tight. Moreover in case $\Psi=\mathbb{R}^{N}$, the assumption that $X$ satisfies the first axiom of countability is superfluous.

Proof. We define a set-valued mapping $\Lambda_{\varepsilon}: X \rightarrow \Psi_{\beta}^{\prime}$ by

$$
\Lambda_{\varepsilon}(x)=\cap_{u \in \Psi}\left\{\eta \in \Psi^{\prime}:|<u, \eta>| \leq p_{\varepsilon}(x, u)\right\}
$$

Then by assumption (c) we have

$$
\lambda(x, \Lambda(x)) \geq 1-\varepsilon
$$

for all $x \in X$ and all $\lambda \in Q$. Therefore we have only to show that $\Lambda_{\varepsilon}$ is compact-valued and upper semicontinuous on $X$.

Compactness. Fix $x \in X$. It is easily verified that $\Lambda_{\varepsilon}(x)$ is a closed, bounded subset of $\Psi_{\sigma}^{\prime}$, and hence it is a compact subset of $\Psi_{\beta}^{\prime}$ by (3) of Proposition 4.

Upper semicontinuity. Let $F$ be a closed subset of $\Psi_{\beta}^{\prime}$ and we show that $\Lambda_{\varepsilon}^{w}(F)=$ $\left\{x \in X: \Lambda_{\varepsilon}(x) \cap F \neq \varnothing\right\}$ is a closed subset of $X$. To do this let $\left\{x_{n}\right\}$ be a sequence of elements of $\Lambda_{\varepsilon}^{w}(F)$ converging to an element $x_{0} \in X$. Then for each $n \geq 1$, we can take an element $\eta_{n} \in F$ with

$$
\left|<u, \eta_{n}>\right| \leq p_{\varepsilon}\left(x_{n}, u\right) \quad \text { for all } \quad u \in \Psi
$$

By assumption (a) we have

$$
\underset{n \rightarrow \infty}{\limsup }\left|<u, \eta_{n}>\right| \leq p_{\varepsilon}\left(x_{0}, u\right) \quad \text { for all } \quad u \in \Psi
$$

and so $\left\{\eta_{n}\right\}$ is weakly bounded. Hence by (3) of Proposition $4,\left\{\eta_{n}\right\}$ has a subsequence which converges in $\Psi_{\beta}^{\prime}$ to some $\eta_{0} \in \Psi^{\prime}$. Then it is readily verified that $\eta_{0} \in F \cap \Lambda_{\varepsilon}\left(x_{0}\right)$ and hence $x_{0} \in \Lambda_{\varepsilon}^{w}(F)$.

Next we assume that $\Psi=\mathbb{R}^{N}$. Then we need only to prove the upper semicontinuity of $\Lambda_{\varepsilon}$. To do this let $\left\{x_{\alpha}\right\}$ be a net of elements of $\Lambda_{\varepsilon}^{w}(F)$ converging to an element $x_{0} \in X$. Then for each $\alpha$, we can take an element $\eta_{\alpha} \in F$ with

$$
\left|<u, \eta_{\alpha}>\right| \leq p_{\varepsilon}\left(x_{\alpha}, u\right) \quad \text { for all } u \in \mathbb{R}^{N} \text {. }
$$


Let $\left\{e_{j}\right\}_{j=1}^{N}$ be a CONS in $\mathbb{R}^{N}$. Then we have

$$
\left\|\eta_{\alpha}\right\|^{2} \leq \sum_{j=1}^{N} p_{\varepsilon}\left(x_{\alpha}, e_{j}\right)^{2}
$$

and hence

$$
\underset{\alpha}{\limsup }\left\|\eta_{\alpha}\right\|^{2} \leq \sum_{j=1}^{N} p_{\varepsilon}\left(x_{0}, e_{j}\right)^{2}<\infty .
$$

Thus we can find a subnet $\left\{\eta_{\alpha^{\prime}}\right\}$ of $\left\{\eta_{\alpha}\right\}$ which is a bounded subset of $\mathbb{R}^{N}$. Therefore there exists a further subnet $\left\{\eta_{\alpha^{\prime \prime}}\right\}$ of $\left\{\eta_{\alpha^{\prime}}\right\}$ converging to some $\eta_{0} \in \mathbb{R}^{N}$. Then $\eta_{0} \in F \cap \Lambda_{\varepsilon}\left(x_{0}\right)$ and the proof is complete.

A. Borel probability measure $\mu$ on $\Psi_{\beta}^{\prime}$ is said to be Gaussian if for each $u \in \Psi$, the function $\eta \in \Psi^{\prime} \mapsto\langle u, \eta\rangle$ is a real (possibly degenerate) Gaussian random variable on the probability measure space $\left(\Psi^{\prime}, \mathcal{B}\left(\Psi_{\beta}^{\prime}\right), \mu\right)$. For a Gaussian Measure $\mu$ on $\Psi_{\beta}^{\prime}$, we define its mean functional $m$ and covariance seminorm $s$ of $\mu$ by

$$
<u, m>=\int_{\Psi^{\prime}}<u, \eta>\mu(d \eta)
$$

and

$$
s(u, v)=\int_{\Psi^{\prime}}<u, \eta-m><v, \eta-m>\mu(d \eta)
$$

for all $u, v \in \Psi$ and we put $s^{2}(u)=s(u, u)$. We know that $m \in \Psi^{\prime}$ and $s$ is a continuous $H$-seminorm on $\Psi$ (see e.g., [5], Theorem 2.6.2).

Let $(X, \mathcal{A})$ be a measurable space. A transition probability $\lambda$ on $X \times \Psi_{\beta}^{\prime}$ is said to be Gaussian if for each $x \in X, \lambda_{x}(\cdot) \equiv \lambda(x, \cdot)$ is a Gaussian measure on $\Psi_{\beta}^{\prime}$. For a Gaussian transition probability $\lambda$ on $X \times \Psi_{\beta}^{\prime}$ we define for each $x \in X$ and each $u, v \in \Psi$,

$$
m(x, u)=\int_{\Psi^{\prime}}<u, \eta>\lambda(x, d \eta)
$$

and

$$
s(x, u, v)=\int_{\Psi^{\prime}}\{\langle u, \eta\rangle-m(x, u)\}\{\langle v, \eta\rangle-m(x, v)\} \lambda(x, d \eta),
$$

and we put $s^{2}(x, u)=s(x, u, u)$. We say that the functions $m: x \in X \mapsto m(x, \cdot)$ and $s: x \in X \mapsto s(x, \cdot, \cdot)$ are the mean function and the covariance function of $\lambda$, respectively. Since a Gaussian measure is uniquely determined by its mean functional and covariance seminorm (see [5], Theorem 2.6.3), it is easily verified that a Gaussian transition probability $\lambda$ is also uniquely determined by its mean function $m$ and covariance function $s$, and hence we write $\lambda=\mathcal{T} \mathcal{N}\left[m, s^{2}\right]$.

The following proposition asserts that a Gaussian transition probability can be characterized in terms of the measurability of its mean and covariance functions. 
Proposition 5. Let $\lambda$ be a mapping from $X$ into $\mathcal{P}\left(\Psi_{\beta}^{\prime}\right)$ such that for each $x \in X, \lambda_{x}$ is a Gaussian measure on $\Psi_{\beta}^{\prime}$ with its mean functional $m(x, \cdot)$ and covariance seminorm $s(x, \cdot, \cdot)$. Then $\lambda$ is a transition probability on $X \times \Psi_{\beta}^{\prime}$ and $\lambda=\mathcal{T N}\left[m, s^{2}\right]$ if and only if for each $u \in \Psi$, the functions $x \in X \mapsto m(x, u)$ and $x \in X \mapsto s^{2}(x, u)$ are measurable.

Proof. For each $u \in \Psi$, we define $\pi_{u}(\eta)=\langle u, \eta\rangle, \eta \in \Psi^{\prime}$. Then we have

$$
\chi\left[\lambda, \pi_{u}\right](x)=\exp \left\{i m(x, u)-\frac{1}{2} s^{2}(x, u)\right\}
$$

and it is easy to see that the function $x \in X \mapsto \chi\left[\lambda, \pi_{u}\right](x)$ is measurable if and only if the functions $x \in X \mapsto m(x, u)$ and $x \in X \mapsto s^{2}(x, u)$ are measurable. Consequently, if we notice that the linear subspace $H=\left\{\pi_{u}: u \in \Psi\right\}$ of $C\left(\Psi_{\beta}^{\prime}\right)$ separates points of $\Psi^{\prime}$, the proof is complete by Proposition 1 since $\Psi_{\beta}^{\prime}$ is a completely regular Suslin space by (2) of Proposition 4.

The following theorem gives a sufficient condition under which a set of Gaussian transition probabilities on $X \times \Psi_{\beta}^{\prime}$ is uniformly tight, in terms of mean and covariance functions.

Theorem 4. Let $X$ be as in Theorem 3 and $Q$ a set of Gaussian transition probabilities on $X \times \Psi_{\beta}^{\prime}$ with $\lambda=\mathcal{T} \mathcal{N}\left[m_{\lambda}, s_{\lambda}^{2}\right], \lambda \in Q$. Assume that there exists a mapping $q: X \times \Psi \rightarrow[0, \infty)$ satisfying $X$;

(a) for each $u \in \Psi$, the mapping $x \in X \mapsto q(x, u)$ is upper semicontinuous on

(b) for each $x \in X, q_{x}(\cdot)=q(x, \cdot)$ is a continuous $H$-seminorm on $\Psi$. Further, assume that there exist non-negative upper semicontinuous functions $M(x)$ and $S(x)$ on $X$ such that for every $x \in X$,

$$
\sup _{\lambda \in Q} q_{x}^{\prime}\left(m_{\lambda}(x)\right) \leq M(x) \quad \text { and } \quad \sup _{\lambda \in Q}\left(s_{\lambda}(x): q_{x}\right)_{H S} \leq S(x) .
$$

Then $Q$ is uniformly tight.

Proof. First we assume that $m_{\lambda}(x)=0$ for all $x \in X$ and $\lambda \in Q$. By assumption and Theorem 2.6.1 of [5] we have

$$
\lambda_{x}\left(\Psi_{q_{x}}^{\prime}\right)=1 \quad \text { for all } \quad x \in X \text { and } \lambda \in Q .
$$

Therefore we may consider that $\lambda_{x}$ is a Gaussian measure on the real separable Hilbert space $\Psi_{q_{x}}^{\prime}$. Thus by Vakhania [13, page 40] we can find a universal constant $K>0$ such that

$$
\lambda_{x}\left(\left\{\eta \in \Psi_{q_{x}}^{\prime}: q_{x}^{\prime}(\eta)>r S(x)\right\}\right) \leq \lambda_{x}\left(\left\{\eta \in \Psi_{q_{x}}^{\prime}: q_{x}^{\prime}(\eta)>r\left(s_{\lambda}(x): q_{x}\right)_{H S}\right\}\right) \leq K e^{-r^{2} / 2}
$$


for all $x \in X, \lambda \in Q$ and $r>0$.

Fix $\varepsilon>0$ and take a constant $r_{\varepsilon}>0$ such that $K e^{-r_{\varepsilon}^{2} / 2}<\varepsilon$. Then we have

$$
\lambda_{x}\left(\left\{\eta \in \Psi_{q_{x}}^{\prime}: q_{x}^{\prime}(\eta)>r_{\varepsilon} S(x)\right\}\right)<\varepsilon
$$

for all $x \in X$ and $\lambda \in Q$. Now we define a set-valued mapping $\Lambda_{\varepsilon}: X \rightarrow \Psi_{\beta}^{\prime}$ by

$$
\Lambda_{\varepsilon}(x)=\left\{\eta \in \Psi^{\prime}:|<u, \eta>| \leq r_{\varepsilon} S(x) q(x, u) \text { for all } u \in \Psi\right\} .
$$

Then we have

$$
\lambda_{x}\left(\Psi^{\prime}-\Lambda_{\varepsilon}(x)\right)<\varepsilon
$$

for all $x \in X$ and $\lambda \in Q$, and $\Lambda_{\varepsilon}$ is compact-valued and upper semicontinuous since $p_{\varepsilon}(x, u) \equiv r_{\varepsilon} S(x) q(x, u)$ satisfies assumptions of Theorem 3 by assumptions (a) and (b).

Next we define for each $\lambda \in Q$,

$$
\gamma_{\lambda}(x, B)=\lambda\left(x, B-m_{\lambda}(x)\right) \quad \text { for all } \quad x \in X \text { and } B \in \mathcal{B}\left(\Psi_{\beta}^{\prime}\right) .
$$

Then by Proposition 5, $\gamma_{\lambda}$ is a Gaussian transition probability with zero mean function and covariance function $s_{\lambda}(x)$. Now we define for each $x \in X$,

$$
\Gamma(x)=\left\{\eta \in \Psi^{\prime}:|<u, \eta\rangle \mid \leq M(x) q(x, u) \text { for all } u \in \Psi\right\}
$$

then by Theorem 3, $\Gamma: X \rightarrow \Psi_{\beta}^{\prime}$ is also compact-valued and upper semicontinuous, and we have

$$
\Lambda_{\varepsilon}(x) \subset \Lambda_{\varepsilon}(x)+\Gamma(x)+m_{\lambda}(x)
$$

for all $x \in X$ and $\lambda \in Q$. Consequently we have

$$
\lambda\left(x, \Lambda_{\varepsilon}(x)+\Gamma(x)\right)=\gamma_{\lambda}\left(x, \Lambda_{\varepsilon}(x)+\Gamma(x)+m_{\lambda}(x)\right) \geq \gamma_{\lambda}\left(x, \Lambda_{\varepsilon}(x)\right) \geq 1-\varepsilon
$$

for all $x \in X$ and $\lambda \in Q$. By (2) of Proposition 2 the mapping $x \in X \mapsto \Lambda_{\varepsilon}(x)+\Gamma(x)$ is compact-valued and upper semicontinuous on $X$, and the proof is complete.

In case $\Psi=\mathbb{R}^{N}$ we have:

Corollary 1. Let $X$ be a topological space and $Q$ a set of Gaussian transition probabilities on $X \times \mathbb{R}^{N}$ with $\lambda=\mathcal{T} \mathcal{N}\left[m_{\lambda}, s_{\lambda}^{2}\right], \lambda \in Q$. Assume that there exist nonnegative functions $M(x, u)$ and $S(x, u)$ defined on $X \times \mathbb{R}^{N}$ which satisfy

(a) for each $u \in \mathbb{R}^{N}$, the functions $x \in X \mapsto M(x, u)$ and $x \in X \mapsto S(x, u)$ are upper semicontinuous on $X$;

(b) $\sup _{\lambda \in Q}\left|<u, m_{\lambda}(x)>\right| \leq M(x, u)$ and $\sup _{\lambda \in Q} s_{\lambda}(x, u) \leq S(x, u)$ for all $x \in X$ and $u \in \mathbb{R}^{N}$.

Then $Q$ is uniformly tight. 
Proof. Let $\left\{e_{i}\right\}_{i=1}^{N}$ be a CONS in $\mathbb{R}^{N}$. Put $q(u, v)=\sum_{j=1}^{N}<u, e_{j}><v, e_{j}>$, $u, v \in \mathbb{R}^{N}, M(x)=\sum_{j=1}^{N} M\left(x, e_{j}\right)$, and $S(x)=\sum_{j=1}^{N} S\left(x, e_{j}\right), x \in X$. Then $q, M$, and $S$ satisfy assumptions of Theorem 4 and the proof is complete.

In the case when $X$ is a one point set we have the following well-known result.

Corollary 2. Let $P$ be a set of Gaussian measures on $\Psi_{\beta}^{\prime}$ with mean functionals $m_{\mu}$ and covariance seminorms $s_{\mu}, \mu \in P$. Assume that $\sup _{\mu \in P}\left|<u, m_{\mu}\right\rangle \mid$ $<\infty$ and $\sup _{\mu \in P} s_{\mu}(u)<\infty$ for each $u \in \Psi$. Then $P$ is uniformly tight.

Proof. Put $p(u)=\sup _{\mu \in P}\left|\left\langle u, m_{\mu}\right\rangle\right|$ and $r(u)=\sup _{\mu \in P} s_{\mu}(u)$ for all $u \in \Psi$. Then $p$ and $r$ are continuous seminorms on $\Psi$ since $\Psi$ is barreled by (1) of Proposition 4 and the $m_{\mu}$ 's and the $s_{\mu}$ 's are continuous. Since $\Psi$ is nuclear we can find a continuous $H$-seminorm $q$ on $\Psi$ such that $\sum_{j=1}^{\infty} p\left(e_{j}\right)^{2}<\infty$ and $\sum_{j=1}^{\infty} r\left(e_{j}\right)^{2}<\infty$ for some $q$-CONS $\left\{e_{j}\right\}_{j=1}^{\infty}$ in $\Psi$. Consequently we have

$$
q^{\prime}\left(m_{\mu}\right)=\left(\sum_{j=1}^{\infty}\left|<e_{j}, m_{\mu}>\right|^{2}\right)^{1 / 2} \leq\left(\sum_{j=1}^{\infty} p\left(e_{j}\right)^{2}\right)^{1 / 2}
$$

and

$$
\left(s_{\mu}: q\right)_{H S}=\left(\sum_{j=1}^{\infty} s_{\mu}\left(e_{j}\right)^{2}\right)^{1 / 2} \leq\left(\sum_{j=1}^{\infty} r\left(e_{j}\right)^{2}\right)^{1 / 2}
$$

Thus $\left\{m_{\mu}\right\}$ and $\left\{s_{\mu}\right\}$ satisfy assumptions of Theorem 4 if we consider $X$ is a one point set, and the proof is complete.

Let $\Phi$ be a nuclear real Fréchet space. In case $X=\Phi_{\beta}^{\prime}$, combined Theorem 1 and Corollary 1 with a well-known criterion for uniform tightness of probability measures on nuclear spaces, we have:

Theorem 5. Let $Q$ be a set of Gaussian transition probabilities on $\Phi_{\beta}^{\prime} \times \Psi_{\beta}^{\prime}$ with $\lambda=\mathcal{T N}\left[m_{\lambda}, s_{\lambda}^{2}\right], \lambda \in Q$. Assume that there exist non-negative functions $M(\xi, u)$ and $S(\xi, u)$ defined on $\Phi^{\prime} \times \Psi$ which satisfy

(a) for each $u \in \Psi$, the functions $\xi \in \Phi_{\beta}^{\prime} \mapsto M(\xi, u)$ and $\xi \in \Phi_{\beta}^{\prime} \mapsto S(\xi, u)$ are upper semicontinuous on $\Phi_{\beta}^{\prime}$; $u \in \Psi$.

(b) $\sup _{\lambda \in Q}\left|m_{\lambda}(\xi, u)\right| \leq M(\xi, u)$ and $\sup _{\lambda \in Q} s_{\lambda}(\xi, u) \leq S(\xi, u)$ for all $\xi \in \Phi^{\prime}$ and Then $P \circ Q$ is uniformly tight for any uniformly tight subset $P$ of $\mathcal{P}\left(\Phi_{\beta}^{\prime}\right)$.

Proof. Before starting to prove the theorem we prepare several notations.

For each $x \in \Phi$ and $u \in \Psi$, let us define mappings as follows:

$$
\pi_{x}(\xi)=<x, \xi>, \quad \xi \in \Phi^{\prime}
$$




$$
\begin{gathered}
\pi_{u}(\eta)=<u, \eta>, \quad \eta \in \Psi^{\prime} \\
\left(\pi_{x} \times \pi_{u}\right)(\xi, \eta)=\left(\pi_{x}(\xi), \pi_{u}(\eta)\right), \quad \xi \in \Phi^{\prime} \quad \text { and } \quad \eta \in \Psi^{\prime} \\
\left(\pi_{x} \times 1\right)(\xi, t)=\left(\pi_{x}(\xi), t\right), \quad \xi \in \Phi^{\prime} \quad \text { and } \quad t \in \mathbb{R} .
\end{gathered}
$$

For each $\gamma \in \mathcal{P}\left(\Phi_{\beta}^{\prime} \times \Psi_{\beta}^{\prime}\right)$ and $\lambda \in \mathcal{T}\left(\Phi_{\beta}^{\prime}, \Psi_{\beta}^{\prime}\right)$, we define a probability measure $\left(\pi_{x} \times \pi_{u}\right) \gamma \in \mathcal{P}\left(\mathbb{R}^{2}\right)$ and a transition probability $\pi_{u} \lambda \in \mathcal{T}\left(\Phi_{\beta}^{\prime}, \mathbb{R}\right)$ by

$$
\begin{gathered}
{\left[\left(\pi_{x} \times \pi_{u}\right) \gamma\right](A \times B)=\gamma\left(\pi_{x}^{-1} A \times \pi_{u}^{-1} B\right) \text { for all } A, B \in \mathcal{B}(\mathbb{R})} \\
{\left[\pi_{u} \lambda\right](\xi, B)=\lambda\left(\xi, \pi_{u}^{-1} B\right) \text { for all } \xi \in \Phi^{\prime} \text { and } B \in \mathcal{B}(\mathbb{R}) .}
\end{gathered}
$$

We now notice that the following uniform tightness criterion is valid: For $A \subset$ $\mathcal{P}\left(\Phi_{\beta}^{\prime} \times \Psi_{\beta}^{\prime}\right)$ to be uniformly tight it is sufficient that for each $x \in \Phi$ and $u \in \Psi$, the set $\left\{\left(\pi_{x} \times \pi_{u}\right) \gamma: \gamma \in A\right\} \subset \mathcal{P}\left(\mathbb{R}^{2}\right)$ is uniformly tight. This result can be proved by investigating the proof of Theorem 3.1 of Mitoma [9], and so we omit the proof. Consequently we prove that for each $x \in \Phi$ and $u \in \Psi$, the set $\left(\pi_{x} \times \pi_{u}\right)(P \circ Q) \subset \mathcal{P}\left(\mathbb{R}^{2}\right)$ is uniformly tight. Since it is easily verified that the equality

$$
\left(\pi_{x} \times \pi_{u}\right)(\mu \circ \lambda)=\left(\pi_{x} \times 1\right)\left(\mu \circ \pi_{u} \lambda\right)
$$

holds and the mapping $\pi_{x} \times 1$ is continuous, we have only to show that the set $P \circ \pi_{u} Q \subset$ $\mathcal{P}\left(\Phi_{\beta}^{\prime} \times \mathbb{R}\right)$ is uniformly tight.

Fix $u \in \Psi$. Since $\pi_{u} \lambda=\mathcal{T} \mathcal{N}\left[m_{\lambda}(\cdot, u), s_{\lambda}^{2}(\cdot, u)\right], \lambda \in Q$, from assumptions and Corollary 1 it follows that $\pi_{u} Q$ is uniformly tight. Thus $P \circ \pi_{u} Q$ is uniformly tight by Theorem 1 since $P$ is uniformly tight.

For the weak convergence of compound probability measures we have:

Theorem 6. Let $\lambda_{\alpha}=\mathcal{T} \mathcal{N}\left[m_{\alpha}, s_{\alpha}^{2}\right]$ be a net of Gaussian transition probabilities on $\Phi_{\beta}^{\prime} \times \Psi_{\beta}^{\prime}$ and $\lambda=\mathcal{T} \mathcal{N}\left[m, s^{2}\right]$ a Gaussian transition probability on $\Phi_{\beta}^{\prime} \times \Psi_{\beta}^{\prime}$. Assume that in addition to assumptions (a) and (b) of Theorem 5,

(c) for each $x \in X$, the sets $\left\{m_{\alpha}(\cdot, u)\right\}$ and $\left\{s_{\alpha}^{2}(\cdot, u)\right\}$ are equicontinuous on every compact subset of $\Phi_{\beta}^{\prime}$;

(d) $\lim _{\alpha} m_{\alpha}(\xi, u)=m(\xi, u)$ and $\lim _{\alpha} s_{\alpha}^{2}(\xi, u)=s^{2}(\xi, u)$ for each $\xi \in \Phi^{\prime}$ and $u \in \Psi$.

Then for any uniformly tight net $\left\{\mu_{\alpha}\right\}$ in $\mathcal{P}\left(\Phi_{\beta}^{\prime}\right)$ converging weakly to $\mu \in$ $\mathcal{P}\left(\Phi_{\beta}^{\prime}\right)$, we have $\mu_{\alpha} \circ \lambda_{\alpha} \stackrel{w}{\rightarrow} \mu \circ \lambda$.

Proof. We first note that $\left\{\mu_{\alpha} \circ \lambda_{\alpha}\right\}$ is uniformly tight by Theorem 5. Fix $u \in \Psi$. Then we have

$$
\chi\left[\lambda_{\alpha}, \pi_{u}\right](\xi)=\exp \left\{i m_{\alpha}(\xi, u)-\frac{1}{2} s_{\alpha}^{2}(\xi, u)\right\} .
$$

and

$$
\chi\left[\lambda, \pi_{u}\right](\xi)=\exp \left\{i m(\xi, u)-\frac{1}{2} s^{2}(\xi, u)\right\} .
$$


Thus from (c) and (d) it follows that

$$
\chi\left[\lambda_{\alpha}, \pi_{u}\right](\xi) \rightarrow \chi\left[\lambda, \pi_{u}\right](\xi) \quad \text { for each } \xi \in \Phi^{\prime}
$$

and the set $\left\{\chi\left[\lambda_{\alpha}, \pi_{u}\right]\right\}$ is equicontinuous on every compact subset of $\Phi_{\beta}^{\prime}$. Moreover, if we set $H=\left\{\pi_{u}: u \in \Psi\right\} \subset C\left(\Psi_{\beta}^{\prime}\right)$ then $H$ separates points of $\Psi^{\prime}$. Consequently we can prove that $\mu_{\alpha} \circ \lambda_{\alpha} \stackrel{w}{\rightarrow} \mu \circ \lambda$ by the same way as in the proof of Theorem 2 .

\section{Acknowledgement}

The author wishes to express his appreciation to the referee for helpful comments and simplifying the original proof of Lemma 1.

\section{References}

[1] R. B. Ash, Real Analysis and Probability, Academic Press, New York, 1972.

[2] I. Csiszár, "Some problems concerning measures on topological spaces and convolutions of measures on topological groups," Les Probabilités sur les Structures Algébriques, Clermont-Ferrand, Colloques Internationaux du CNRS, Paris, 75-96, 1969.

[3] I. Csiszár, "On the weak* continuity of convolution in a convolution algebra over an arbitrary topological group," Studia Sci. Math. Hungar., 6, 27-40, 1971.

[4] X. Fernique, "Processus linéaires, processus généralisés," Ann. Inst. Fourier, Grenoble, 17, 1-92, 1967.

[5] K. Itô, "Foundations of Stochastic Differential Equations in Infinite Dimensional Spaces," CBMS 47, Society for Industrial and Applied Mathematics, Philadelphia, 1984.

[6] G. Kallianpur, "The topology of weak convergence of probability measures," J. Math. Mech., 10, 947-969, 1961.

[7] J. Kawabe, "Convergence of compound probability measures on topological spaces," Colloq. Math., 67, 161-176, 1994.

[8] E. Klein and A. C. Thompson, Theory of Correspondences, Wiley, New York, 1984.

[9] I. Mitoma, "Tightness of probabilities on $C\left([0,1] ; \varphi^{\prime}\right)$ and $D\left([0,1] ; \varphi^{\prime}\right)$," Ann. Probab., 11, 989-999, 1983.

[10] Yu. V. Prokhorov, "Convergence of random processes and limit theorems in probability theory," Theory Probab. Appl., 1, 157-214, 1956.

[11] H. H. Schaefer, Topological Vector Spaces, Springer, New York, 1971.

[12] L. Schwartz, Radon Measures on Arbitrary Topological Spaces and Cylindrical Measures, Oxford University Press, 1973.

[13] N. N. Vakhania, Probability Distributions on Linear Spaces, North Holland, New York, 1981.

[14] N. N. Vakhania, V. I. Tarieladze and S. A. Chobanyan, Probability Distributions on Banach Spaces, D. Reidel Publishing Company, 1987.

[15] K. Yosida, Functional Analysis (Sixth edition), Springer, New York, 1980.

Department of Mathematics, Faculty of Engineering, Shinshu University, Wakasato, Nagano 380, Japan. E-mail address: jkawabe@gipwc.shinshu-u.ac.jp 